\title{
CÁNCER DE TIROIDES EN NÓDULO HIPERCAPTANTE POR GAMMAGRAFÍA. SERIE DE CASOS.
}

\author{
Diana F. Rozo, $M D^{1 *}$, James Yurgaky, MD ${ }^{1}$, Diana Polanía, $M D^{2}$, \\ Jorge García, $M D^{2}$ Y Carlos García, $M D^{2}$ \\ ${ }^{1}$ Residente del programa de Endocrinología, Facultad de Medicina, Universidad Militar Nueva Granada, \\ Bogotá. ${ }^{2}$ Endocrinólogo, Servicio de Endocrinología, Hospital Militar Central, Bogotá.
}

\begin{abstract}
Resumen
La presencia de nódulos tiroideos es una condición clínica de alta prevalencia en la población general. Cuando gammagráficamente son hipercaptantes a menudo se considera como una condición benigna; sin embargo, a pesar de presentarse esta condición, cuando la clínica es sugestiva de malignidad, es recomendado la realización de un estudio histológico. En este artículo se presentan tres casos en los que la clínica nos hizo sospechar de malignidad y se confirmó con estudios histológicos posteriores.
\end{abstract}

Palabras clave: cáncer de tiroides, nódulo tiroideo, aumento de captación

\section{THYROID CANCER IN INCREASED UPTAKE NODULE BY GAMMAGRAPHY. SERIES OF CASES}

\begin{abstract}
The presence of thyroid nodules is a clinical condition of high prevalence in the general population. When thyroid nodule gammagraphy shows increased uptake, it is usually considered benign; however this condition is given and when clinical study suggests malignancy, a histological study is recommended. This article present three patients that in spite of an increased uptake, their clinical characteristics suggested the possibility of malignancy, situation that was later confirmed with histological studies.
\end{abstract}

Key words: thyroid cancer, thyroid nodule, increase uptake

\section{CÂNCER DE TIREÓIDE EM NÓDULO HIPERCAPTANTE POR GAMAGRAFIA. SÉRIE DE CASOS}

\begin{abstract}
Resumo
A presença de nódulos na tireóide é uma condição clínica com alta prevalência na população geral. Quando pela gamagrafia são hipercaptantes muitas vezes é considerada uma condição benigna; no entanto, apesar desta condição, quando a clínica é sugestiva de malignidade, é recomendável a realização de um estudo histológico. Este artigo apresenta três casos em que a clínica nos fez suspeitar de malignidade e se confirmaram pela histologia subseqüente.
\end{abstract}

Palabras-chave: neoplasias da glândula tireóide, nódulo da glândula tireóide, aumento da captação

* Correspondencia: Diana Rozo, drozomaragua@gmail.com. Dirección postal: Servicio de Endocrinología, Hospital Militar Central, Tr. 3 No 40-00, Tel 3486868 ext: 5060, Bogotá, Colombia.

Recibido: Octubre 25 de 2010 Aceptado: Diciembre 15 de 2010 


\section{Introducción}

El nódulo tiroideo es una enfermedad extremadamente frecuente, el porcentaje de detección de esta enfermedad depende del método utilizado para la evaluación, los cuales pueden llegar a ser tan altos como el $67 \%$ reportado en algunos estudios (1,2). La gran preocupación en relación a esta enfermedad siempre ha sido establecer su potencial de malignidad, ya que este factor es uno de los principales determinantes en la escogencia del tratamiento y por lo consiguiente en su pronóstico. Como en cualquier patología, la clínica es la clave para llegar a un diagnóstico exitoso, y así cuando nos enfrentamos en el examen físico, a la presencia de un nódulo tiroideo con características sospechosas de malignidad, es importante para sustentar el diagnóstico, hacer un estudio ecográfico y patológico para estudiar la histología.

El uso de la gammagrafía tiroidea tiene claras indicaciones como lo son la presencia de bocio multinodular, evaluación de nódulo hiperfuncionante cuando existe la sospecha y seguimiento de lesión folicular de bajo grado.

En este artículo se presentan tres casos en los cuales a pesar de observarse en la gammagrafía un nódulo tiroideo hipercaptante, su manejo fue diferente, por la alta sospecha clínica de ser un carcinoma de tiroides, lo cual no es frecuente. Es importante anotar que para un diagnóstico rápido, preciso y un tratamiento efectivo se requiere de un examen físico minucioso.

\section{Caso 1}

Mujer de 46 años remitida por bocio de cinco años de evolución sin referir síntomas compresivos, ni dolor y a veces taquicardia en reposo. Revisión por sistemas: Xerodermia y caída del pelo.

Antecedentes patológicos: dislipidemia, asma leve, enfermedad ácido péptica y síndrome de intestino irritable. Quirúrgicos: cistopexia. Farmacológicos: lansoprazol, atorvastatina. Ginecológicos: G2P2V2. Familiares: madre con hipotiroidismo.

Exámen físico: buen estado general con tensión arterial (TA): 120/80, FC: 80 , peso: $57 \mathrm{~kg}$, talla: $1,50 \mathrm{cms}$, IMC: 23. Se palpa tiroides grado 2 , dominancia izquierda, consistencia dura. La paciente trae a la consulta los siguientes resultados de laboratorio: TSH: $1,46 \mathrm{u} / \mathrm{l}, \mathrm{T} 4$ total: $7,45 \mathrm{mg} / \mathrm{dl}$ (valor referencia: $5-14 \mathrm{mg} / \mathrm{dl}$ ) y T3 total: $0,93 \mathrm{nmol} / \mathrm{L}$ (valor referencia $0-0,82$ )
Se le realiza gammagrafía de tiroides en donde se evidencia nódulo hipercaptante en lóbulo izquierdo que no suprime en su totalidad el resto de la glándula con un índice de atrapamiento de 4,8 (Figura 1).

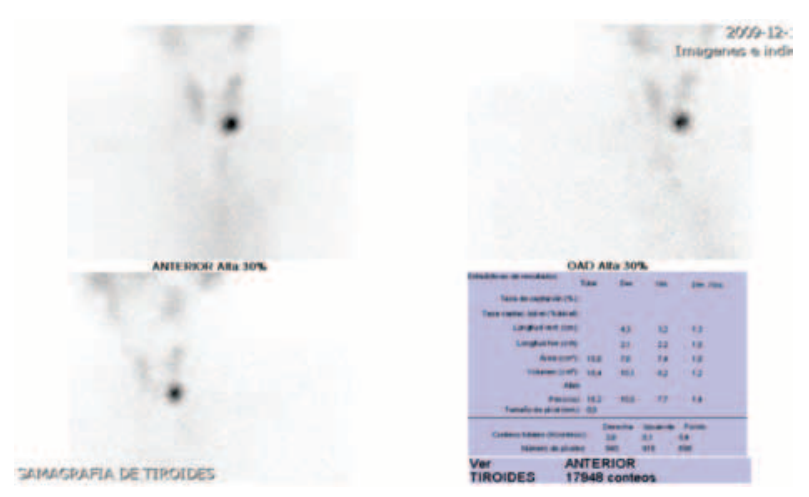

Figura 1. Gammagrafía de tiroides.

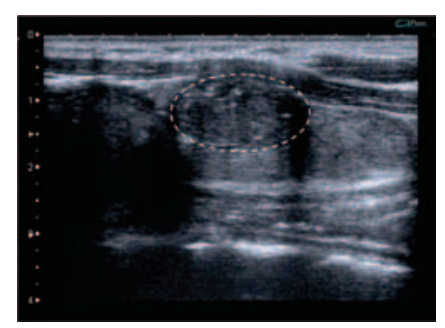

Figura 2. Ecografía de tiroides. Nódulo en lóbulo izquierdo de 22 * 11 mm, sólido, hipoecóico, micro-calcificaciones en su interior, bien delimitado y que corresponde a zona hipercaptante de gamagrafía.

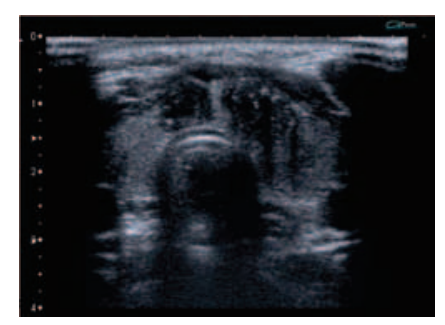

Figura 3. Ecografía de tiroides. Nódulo de $9 \mathrm{~mm}$ en istmo

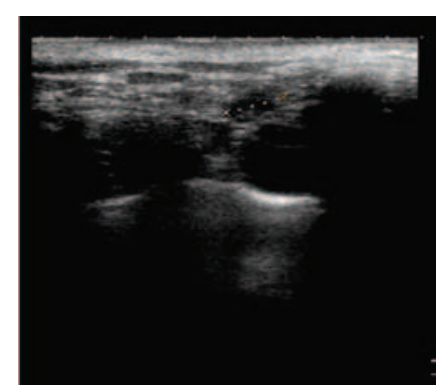

Figura 4. Ecografía de tiroides Ganglios hipoecóicos que sugieren metástasis ubicados en región paratraqueal derecha, supra y retrocalvicular derecha, y periyugular ipsilateral) 
Por dureza a la palpación de lóbulo izquierdo y los hallazgos en la ecografía de tiroides (Figuras 2, 3 y 4), se decide la realización de biopsia aspirativa con aguja fina (BACAF) de tiroides, de la cual se reporta abundantes grupos de células foliculares con inclusiones intranucleares y núcleos en grano de café sugestiva de cáncer papilar.

A la paciente se le realiza tiroidectomía total y vaciamiento ganglionar. La tiroides tenía un peso 20 gramos, un tamaño de $6 \times 4 \times 1,5 \mathrm{cms}$, el lóbulo derecho $4 \times 2 \times 1,5 \mathrm{cms}$ y lóbulo izquierdo $4 \times 2 \times 2 \mathrm{cms}$. En el istmo y lóbulo izquierdo hay una lesión blanquecina circunscrita de $3 \times 2 \times 2 \mathrm{cms}$.

Basados en la patología el diagnóstico fue carcinoma papilar de tiroides variante de célula alta, observándose a nivel del istmo y lóbulo izquierdo de $3 \mathrm{cms}$ en diámetro mayor y foco microscópico de $0,1 \mathrm{~cm}$ en el lóbulo derecho, con infiltración focal de tejidos peritiroideos por tumor sin compromiso de márgenes de resección, con evidencia de cinco ganglios positivos (mediastino superior \#2, periyugular \#2, peritiroideo \#1) borde de resección del tumor libres, 2 paratiroides y tiroiditis de Hashimoto (Figuras 5 y 6 ).

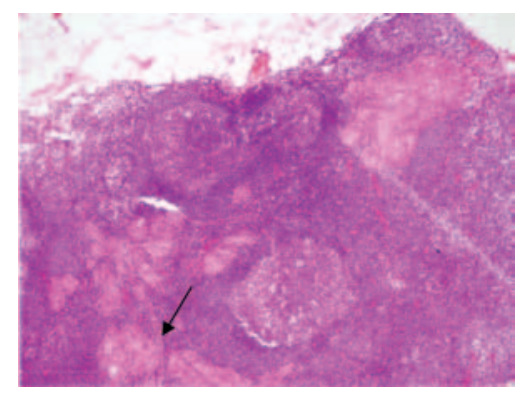

A

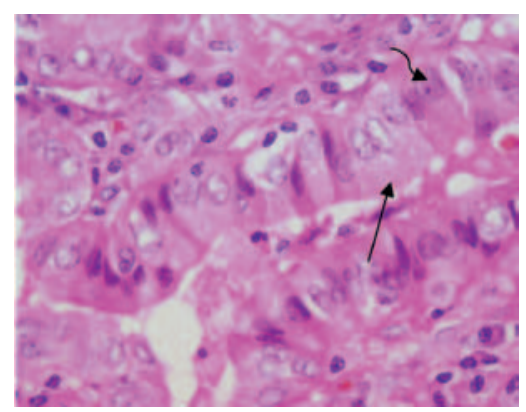

B

Figura 5. Corte de tiroides coloreado con hematoxilina-eosina. A: tejido metastásico en tejido tiroideo (flecha). B: células altas con gran citoplasma (flecha recta) y célula en gránulo de café (flecha curva).

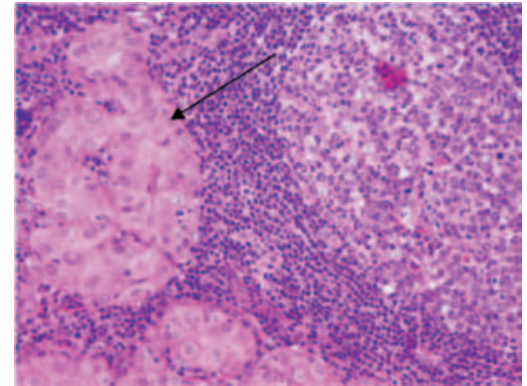

Figura 6: Corte de tiroides coloreado con hematoxilina-eosina: ganglio con metástasis en tejido tiroideo.

Se estadifica con MACIS: 4,98 con riesgo $1 \%$.

Se le administra $\mathrm{I}^{131}$ (I: yodo), se hace seguimiento clínico y se le realizan pruebas bioquímicas con los siguientes resultados: TSH: $0,2 \mathrm{u} / \mathrm{l}$, anticuerpos antitiroglobulina: negativos $(<20 \mathrm{UI} / \mathrm{ml})$, tiroglobulina: $0,1 \mathrm{ng} / \mathrm{ml}$ y en el control de ecografía de cuello no hay evidencia de adenopatías.

\section{Caso 2}

Mujer de 42 años quien consulta por cuadro de disfonía de dos meses de evolución sin otro síntoma asociado. Antecedentes médicos negativos, quirúrgicos: histerectomía a los 40 años por miomas. Niega exposición a tóxicos, o alergias conocidas, sin antecedentes familiares.

Examen físico: buen estado general, TA: 120/70, FC: 72, FR: 16, IMC: 25, conjuntivas normocrómicas y en palpación de cuello hay aumento de tamaño de tiroides con palpación de nódulo duro y móvil de $1 \mathrm{x}$ $1 \mathrm{cms}$ aproximadamente en polo superior de lóbulo derecho. La paciente trae a la consulta el reporte de una gammagrafía de tiroides en la cual se evidencia en el lóbulo derecho un nódulo hipercaptante con un índice de 5,1 y con TSH: 3,1 mU/L. Sin embargo, además de la disfonía de dos meses de evolución que la paciente presentaba y no explicada en ese momento, llamaba la atención la consistencia dura del nódulo tiroideo a la palpación.

Por los hallazgos clínicos se decidió realizar una ecografía de tiroides como ayuda diagnóstica, para descartar la presencia de malignidad; el resultado reporta la presencia de un nódulo sólido de $8 \times 6 \mathrm{~mm}$ en el polo superior del lóbulo derecho, razón por lo cual se le realiza BACAF de tiroides en donde se pueden 
observar células foliculares y papilas con inclusiones intranucleares, linfocitos normales con histiocitos multinucleados, hecho que hace pensar en cáncer papilar y tiroiditis linfocítica.

Es llevada a intervención quirúrgica donde encuentran nódulo de $5 \mathrm{~mm}$ en lóbulo derecho con invasión capsular macroscópica y en lóbulo izquierdo evidencia de fibrosis en tercio medio, con intensa reacción fibrótica que compromete el laríngeo recurrente y la presencia de adenopatías, las cuales por estudios de biopsia por congelación no revelaron ningún hallazgo de malignidad. Se realiza tiroidectomía total más vaciamiento ganglionar. El reporte de patología mostró: glándula tiroides de 16,2 gramos, cáncer papilar variedad folicular de $0,5 \mathrm{cms}$ con compromiso de cápsula únicamente, ganglios negativos y tejido peritiroideo sin paratiroides.

La paciente evoluciona satisfactoriamente en el postoperatorio inmediato; después de la tiroidectomía total se le administraron 100 mci de $\mathrm{I}^{131}$. Los resultados de los exámenes tomados fueron: tiroglobulinas: $<0,1 \mathrm{ng} /$ $\mathrm{ml}$, anticuerpos anti-tiroglobulinas: negativos (<20 UI/ $\mathrm{ml}$ ). Los valores altos de TSH fueron suprimidos con el tratamiento con levotiroxina 250 ug al día. A los dos años, hubo aumento de la tiroglobulina a $1,8 \mathrm{ng} / \mathrm{ml}$ a pesar de terapia supresora. Se le realizó rastreo el cual fue positivo por captación en remanente tiroideo; por lo tanto, se le administró nuevamente 150 mci de ${ }^{131}$, para una dosis acumulada de $250 \mathrm{mci}$. Se le hicieron estudios controles adicionales como la ecografía de cuello, en la cual no se evidenció la presencia de adenopatías.

La paciente evolucionó satisfactoriamente y los resultados de los exámenes de control fueron: tiroglobulina: $<0,1, \mathrm{TSH}: 0,03 \mathrm{mU} / \mathrm{L}$, anticuerpos anti-tiroglobulina: negativos, calcio sérico: $9,5 \mathrm{mg} / \mathrm{dl}$, albúmina: $4,5 \mathrm{gr} /$ dl y ecografía de tiroides en estado post tiroidectomía sin adenopatías. Se consideró cáncer de tiroides de bajo riesgo, MACIS de 4 con riesgo de $1 \%$ y se decide continuar con el seguimiento.

Aunque el nervio laríngeo recurrente estaba comprometido por fibrosis, se consideró la valoración por el servicio de otorrinolaringología para el estudio y manejo de la disfonía, quienes realizaron telelaringoscopia en la cual se observó parálisis de cuerda vocal derecha, por lo que se sometió a tratamiento de terapia vocal.

\section{Caso 3}

Mujer de 54 años remitida a la consulta de endocrinología por síntomas de astenia, adinamia, somnolencia, caída del pelo y xerodermia sin síntomas compresivos. Los antecedentes médicos fueron: hemorragia uterina anormal, dislipidemia y enfermedad ácido péptica. El resto de los antecedentes fueron negativos.

Examen físico: TA: 120/80, FC: 70, FR: 16, IMC: 22, talla: $1,54 \mathrm{~cm}$, peso: $55 \mathrm{~kg}$, a la palpación en cuello se encuentra la tiroides disminuida de tamaño y se evidencia, nódulo izquierdo de $0,5 \mathrm{~cm}$ de consistencia dura. La paciente trae a la consulta un reporte de los siguientes laboratorios: TSH: 7,27 mU/L, T4 libre: 1,25 , y una gamagrafía de tiroides en donde se puede observar nódulo hipercaptante en lóbulo derecho.

Se inicia el tratamiento con levotiroxina 50 ug al día; debido al hallazgo encontrado en el examen físico, se decide la realización de una ecografía de tiroides, en la cual se evidencia en el en lóbulo izquierdo, nódulo sólido central de 9x6×5 mm con calcificaciones irregulares con vascularización; en el polo inferior del mismo lóbulo se observó nódulo de $3 \mathrm{~mm}$ con dos adenopatías de 6 y $4 \mathrm{~mm}$ en zona IV de cuello, con características sospechosas. Por lo anterior se realiza $\mathrm{BACAF}$ de tiroides con la que se evidencian células foliculares mal preservadas, células microfoliculares de tamaños variables y coloide que hace pensar en una lesión folicular que favorece la formación de un nódulo adenomatoso.

La paciente es llevada a intervención quirúrgica, y la patología ordenada reporta cáncer papilar de $0,3 \mathrm{~cm}$ de variedad folicular sin invasión capsular en lóbulo izquierdo; el lóbulo derecho se reporta libre de enfermedad, pero se presentan dos ganglios positivos para metástasis. La paciente evoluciona satisfactoriamente y en el momento posterior a tiroidectomía total se le administran 100 mci de I ${ }^{131}$; se le realizaron exámenes de control y los resultados obtenidos fueron: tiroglobulina: $0,2 \mathrm{ng} / \mathrm{ml}$, anticuerpos anti-tiroglobulina: negativos, TSH: $0,1 \mathrm{mU} / \mathrm{L}$ (valores de referencia: 0,5-4 mU/L), calcio sérico: $9,1 \mathrm{mg} / \mathrm{dl}$ y fósforo: $3,76 \mathrm{mg} / \mathrm{dl}$. Se continúa seguimiento de la paciente, y se considera dado los hallazgos previamente descritos en los exámenes control que la paciente se encuentra en remisión de su enfermedad con MACIS de 4,3 y riesgo de $1 \%$. 


\section{Discusión}

La presencia de nódulos en la tiroides no necesariamente indican malignidad, sin embargo cuando coexisten algunos factores como la edad (si es $<20$ años y mayor de 70 años), historia de radiación previa , historia familiar de cáncer tiroideo, antecedente de enfermedad tiroidea autoinmune y la presencia síntomas de hiper o hipotiroidismo, ayudan a guiar el estudio de la lesión (3).

Además de los antecedentes médicos y familiares que se describieron previamente, se debe tener en cuenta la presencia de algunas características que obligan al estudio complementario de los nódulos por medio de un análisis histológico; entre ellas están la presencia de síntomas obstructivos de la vía aérea superior, sensación de disnea, tos, parálisis de cuerdas vocales, disfonía o voz gruesa no explicada y/o al examen físico, la presencia a la palpación de un nódulo tiroideo de consistencia dura con o sin la presencia de adenopatía cervical sobre todo si es ipsilateral y de rápido crecimiento. Entre las características imagenológicas observadas en la ecografía está la presencia de hipoecogenicidad, micro calcificaciones, bordes irregulares, aspecto sólido, vascularización central, ausencia de halo periférico y en lo relacionado con el tamaño, si es más alto que ancho y esa diferencia es mayor de $1 \mathrm{~cm}$ (3).

A pesar de ser ampliamente aceptado, que el riesgo de malignidad de un paciente que tenga TSH suprimida y que en la gammagrafía se observe un nódulo hipercaptante, es inferior al $10 \%$ (5), se encuentra en la literatura reportes de casos de pacientes con las características descritas previamente y presencia de malignidad por tal razón, el clínico debe estar alerta y continuar con los estudios que ayuden a detectar si existe la malignidad en el nódulo, en los casos en que se han detectado (6).

El valor diagnóstico de la gammagrafía tiroidea para el estudio de nódulos tiroideos es limitado; es una técnica de medicina nuclear que usa diferentes radiotrazadores como I ${ }^{123}, \mathrm{I}^{131}$ o Tecnesio ${ }^{99}$ (4). Típicamente el nódulo se caracteriza por su capacidad de captar el radiotrazador y dependiendo de esta capacidad se determina si el nódulo es caliente (captación elevada) o frío (captación disminuida); en estos últimos la posibilidad de cáncer es alrededor del $15 \%$ por lo que es necesario e imperativo el estudio de BACAF (7). Un estudio recientemente publicado en Brasil (4), en el cual 102 pacientes que tenían un nódulo tiroideo, se les realizó BACAF. Aquellos que por análisis histológico tenían un diagnóstico de indeterminado o Bethesda I, se les realizó una gamagrafía con $\mathrm{I}^{123}$, y se pudo evidenciar que la presencia de un nódulo caliente tenía una correlación de $2.5 \%$ vs $25 \%$ si el nódulo fue clasificado inicialmente como frío.

En el estudio de nódulo tiroideo la gammagrafía tiroidea es útil en los siguientes casos (8):

- Para seleccionar a los pacientes con bocio multinodular que pueden ser candidatos para el estudio histológico por BACAF.

- Para evaluar la posibilidad de que un nódulo sea hiperactivo o hiperfuncionante.

- Para decidir si se hace seguimiento de lesiones foliculares de bajo grado.

También es útil si se tiene el resultado de un análisis histológico que no da un diagnóstico, o a una lesión folicular de bajo grado, la gammagrafía ayuda a la discriminación entre nódulo caliente o frío y por lo tanto, para decidir entre la conducta quirúrgica vs. el seguimiento $(9,10)$. En el caso de un hipertiroidismo documentado mediante una TSH suprimida es extremadamente útil ya que el riesgo de malignidad es menor al $1 \%$ (11).

En nuestro medio la gammagrafía se realiza con tecnesio ${ }^{99}$ debido a su disponibilidad; por tal razón, los datos disponibles en la literatura de pacientes valorados con $I^{123}$ no se pueden extrapolar debido a que el tecnecio no se organifica en la glándula tiroidea y por lo tanto, se pueden encontrar diferencias entre estas dos técnicas; entonces, de los resultados obtenidos por los dos métodos de diagnóstico, no es posible hacer una equivalencia del $100 \%$ (15).

Cakir y col. (13) reportaron en pacientes con hipertiroidismo, la presencia de malignidad en un $6,9 \%$; es importante entonces diferenciar entre aquellos pacientes que tienen hipertiroidismo patente vs subclínico ya que para el primer grupo, el riesgo de malignidad es menor al $1 \%$ mientras que para el grupo de hipertiroidismo subclínico este valor es del 3-5 \% (14).

La mayoría de los cánceres asociados a nódulos hipercaptantes son diferenciados, $80 \%$ son papilares y el $10 \%$ son foliculares (16). La coexistencia de nódulos hiperfuncionantes y cáncer puede explicarse en 
la mayoría de los casos, por la presencia en estrecha relación, de un foco tiroideo funcionante con la lesión neoplásica y en la minoría de los casos, se trata de un real hiperfuncionamiento del cáncer per se (17); es importante anotar que en presencia de hipertiroidismo, la probabilidad de malignidad del nódulo hipercaptante detectado por gammagrafía es inferior al $1 \%$. La agudeza clínica del examinador apoyada en los resultados de otras imágenes como las de la ecografía cuando es necesario, pueden ayudar al diagnóstico de una enfermedad neoplásica (18).

Finalmente, en los casos presentados en este artículo, a pesar de los resultados de los análisis paraclínicos y que los imagenológicos (gammagrafía de tiroides) mostraban un nódulo tiroideo que orientaban hacia un diagnóstico de enfermedad benigna, ciertas características detectadas al realizar el examen físico y la sintomatología observada llevó a sospechar la presencia de malignidad, e indujo que el médico tratante hiciera una búsqueda exhaustiva, para descartar en el nódulo tiroideo observado, la presencia de una neoplasia maligna.

\section{Conclusiones}

El nódulo tiroideo es una enfermedad prevalente en la población general, con una frecuencia de detección variable que depende del método usado para su diagnóstico. Si bien es cierto que características paraclínicas como la presencia en la ultrasonografía de tiroides de un nódulo con cualquiera de las siguientes características: hipoecogénico, micro calcificaciones, bordes irregulares, aspecto sólido, vascularización central, ausencia de halo periférico y en lo relacionado con el tamaño, si es más alto que ancho y esa diferencia es mayor de $1 \mathrm{~cm}$, al igual que el hiperfuncionamiento expresado como supresión de hormona estimulante de tiroides e incremento de tiroxina circulante, y los hallazgos en la gammagrafía de captación alta o baja, son ayudas absolutamente trascendentales en el momento de afrontar el estudio de un paciente con enfermedad tiroidea nodular. También es importante la experiencia del clínico para identificar mediante la anamnesis y el examen físico, características sospechosas de malignidad tales como la consistencia tiroidea pétrea, adenomegalia ipsilateral, cambios en las características de la voz y rápido crecimiento de la lesión, signos que son muy valiosos para orientar al médico para que inicie los estudios complementarios histotológicos en los pacientes que los requieran y así tener un diagnóstico adecuado.

\section{Referencias}

1. Bui A, Mazzaferri E L. New Paradigms in the Diagnosis and Management of Thyroid Nodules. The Endocrinologist. 2007;17(1):35-45.

2. Tan G H, Gharib H. Thyroid Incidentalomas: Management Approaches to Nonpalpable Nodules Discovered Incidentally on Thyroid Imaging. Ann Intern Med. 1997;126:226-231.

3. Bommireddipalli S. Follicular Variant of Papillary Thyroid Carcinoma Presenting as a Toxic Nodule by I-123 Scintigraphy , Clinical Nuclear Medicine. 2010;35(10):770-5.

4. Leenhardt L, Bernier M O, Boin-Pineau M H. Advances in Diagnostic Practices Affect Thyroid Cancer Incidence in France. Eur J Endocrinol. 2004;150:133-9.

5. Rosário P W. Contribution of Scintigraphy and Ultrasonography to the Prediction of Malignancy in Thyroid Nodules with Indeterminate Cytology. Arq Bras Endocrinol Metab. 2010;54(1):56-9.

6. Ashcraft M W, Van Herle A J. Management of Thyroid Nodules. II. Scanning Techniques, Thyroid Suppressive Therapy, and Fine Needle Aspiration. Head Neck Surg. 1981;3:297-322.

7. Niepomniszcze H, Suarez H, Pitoia F. Case Reports: Follicular Carcinoma Presenting as Autonomous Functioning Thyroid Nodule and Containing an Activating Mutation of the TSH Receptor (T620I) and a Mutation of the Ki-RAS (G12C) genes. Thyroid. 2006;16:497-503.

8. Ross D S. Diagnostic Approach to and Treatment of Thyroid Nodules. I. In: Rose B D, ed. UpToDate. Wellesley, M A: UpToDate. Last accessed December 2008.

9. Marc D C. Evaluation and Imaging of a Thyroid Nodule. Surg Oncol Clin N Am. 2008; 17:37-56.

10. Singer P A, Cooper D S, Daniels G H. Treatment Guidelines for Patients with Thyroid Nodules and Well-differentiated Thyroid Cancer. Arch Intern Med. 1996;156:2165-72.

11. Mazzaferri E L. Management of a Thyroid Solitary Nodule. N Engl J Med. 1993;328:553-9.

12. Wong C K, Wheeler M H. Thyroid Nodules: Rational Management. World J Surg. 2000;24:934-41.

13. Cakir M, Arici C, Alakus H. Incidental Thyroid Carcinoma in Thyrotoxic Patients Treated by Surgery. Horm Res. 2007;67:96-9.

14. McCaffrey T V. Evaluation of the Thyroid Nodule. Cancer Control. 2000;7:223-8.

15. Cooper D S, Doherty G M, Haugen B R. Management Guidelines for Patients with Thyroid Nodules and Differentiated Thyroid Cancer: The American Thyroid Association Guidelines Taskforce. Thyroid. 2006;16:109-42.

16. Ragni F, Pinelli D, Facchini M. Thyroid Carcinoma in Hyperthyroid Syndromes in Italian. G Chir. 1996;17:158-165.

17. Meller J, Becker W. The Continuing Importance of Thyroid Scintigraphy in the Era of High-resolution Ultrasound. Eur $J$ Nucl Med Mol Imaging. 2002;29(2):425-38.

18. Horvath E, Majlis S, Rossi R. An Ultrasonogram Reporting System for Thyroid Nodules Stratifying Cancer Risk for Clinical Management. J Clin Endocrinol Metab. 2009;94:1748-51. 